\title{
CORRESPONDENCE
}

('The following contribution to $\mathrm{Mr}$. North's lecture was inadvertertly not printed in the July Journal, and is here printed in the form of correspondence.)

\section{Townend Rings on Pusher Engines}

To the Editor of the Journal of the Royal Aeronautical Society.

Dear Sir,-I should be glad if Mr. North would add whatever facts of a quanlitative nature he can to his remarks under the above heading. It would appear to me that, apart from the possible increase in cooling, the reduction in drag would result only from maintaining a non-turbulent air flow on the body immediately ahead of the ring. If this conception is correct, then I should expect the reduction in drag to be very small indeed, say, of the order of five per cent. I should expect the performance of the pusher airscrew to be noticeably improved by the action of the ring and should be glad if Mr. North would confirm this, or otherwise.

Townend Rings.-Because the ring acts as an aerofoil, its attitude on tractor machines gives it a considerable forward force component under high speed conditions (250 m.p.h. and over). Also, I understand from the N.A.C.A. tests that the fore and aft position is not sensitive to positions of $4^{\prime \prime}-5^{\prime \prime}$ on a $600 \mathrm{~h} . \mathrm{p}$. radial engine. Hence, it has occurred to me that here we have a source of energy which, so far as I know, has hitherto not been tapped, for, if the ring were suitably mounted on slides, or linkages, one could begin with the ring in a back position and allow the forward travel to do work, when the ring was allowed to move forward. For the ring under consideration, I should estimate the energy available would be of the order of $4,000-5,000$ lbs. ins., which might be utilised for assisting to retract the undercarriage or for automatically controlling wing flaps and the like. I should be glad to know Mr. North's opinion on the practicability of such an application.

Yours faithfully,

\section{F. RADCLIFFE.}

Dear Sir,-In answer to Mr. Radcliffe, the figure of 40 per cent. reduction on a pusher nacelle has already been quoted, and probably much better results could be obtained with a nine-cylinder engine and a careful study of the best form of nacelle shape. The ring has the effect of correcting the divergent air stream which these cylinders tend to set up. I am not aware of any experimental results on the effect on performance of pusher airscrews, but I am doubtful if there are any general grounds for expecting an abnormal increase in nett propulsive efficiency. Mr. Radcliffe's proposal for using a ring as a source of energy is ingenious, but it appears to me that the practical disadvantages and complications of the mechanism involved would make one hesitate in attempting to make use of this energy.

Yours faithfully,

J. D. NORTH. 